\title{
The Role of Liver Transplantation in the Treatment of Hepatocellular Carcinoma - a Single Center Experience from Budapest
}

\author{
Piros L, Görög D, Fehérvári I, Nemes B, Langer R, Kóbori L \\ Department of Transplantation and Surgery, Faculty of Medicine, Semmelweis University, Budapest, Hungary
}

\begin{abstract}
Background: Liver transplantation for hepatic tumours is known as a proven treatment opportunity. The outcome has improved over the past decades because of the appropriate recipient selection.

Materials and methods: In a single-institution study of 29 consecutive adult patients underwent orthotopic liver transplantation (OLT) between 1 January 2004 and 1 January 2011 in the Semmelweis University, Budapest with the indication of hepatocellular carcinoma (HCC).

Results: The 1-year survival was $85.7 \%$ and the 3-year survival was $71.7 \%$.

Conclusions: Further investigations are needed on the field of histological and biological attributes of the hepatocellular carcinoma. Our aim is to find a better and individualized immunosuppressive protocol, which is also protective against malignant tumours.
\end{abstract}

Keywords: hepatocellular carcinoma, liver transplantation, mTOR-inhibitor

Received: 20 June 2011

\section{Introduction}

Liver transplantation (OLT) is a well-established and durable surgical therapy for all forms of end-stage liver disease and for certain malignancies. Over the years, the liver transplant survival rate improved, Adam R. et al. [1] descibed a 1 - and 5-year survival of $83 \%$ and $75 \%$, respectively. The success of such treatment has resulted in a progressively increasing claim for liver transplantation. At the same time, the organ donor shortage is rising. Several strategies have been established to increase the number of liver transplantation, such as harvesting organs from nonheart-beating donors and live donors, liver splitting and using organs from expanded criteria donors.

Liver transplantation is the best treatment option for patients with hepatocellular carcinoma and liver cirrhosis, unless they have excellent liver function and minimal portal hypertension [2]. Early experience of liver transplantation for patients with hepatocellular carcinoma was poor because tumoral recurrence was very frequent [3]. In 1996, Mazzaferro et al. [4] showed that a subgroup of patients with radiologic evidence of a single tumor $\leq 5 \mathrm{~cm}$ in diameter, or two to three tumors $\leq 3 \mathrm{~cm}$ in diameter had 5-year and recurrence-free survival rates of 75 and $83 \%$, respectively. The Milan criteria were subsequently adopted by the United Network for Organ Sharing (UNOS) staging system for allocating organs for liver transplantation in the United States. However, recent data suggest that Milan criteria may be too conservative. Yao et al. [5] reported their results with a moderate expansion of these criteria (the so-

Correspondence to: László Piros

E-mail: pirosl666@hotmail.com called University of California at San Francisco (UCSF) criteria). Patients selected with these expanded criteria had disease-free survival that was comparable to survival of patients selected with Milan criteria.

In Hungary the first liver transplantations were performed in 1995 in the Department of Transplantation and Surgery of the Semmelweis University, Budapest. In the case of hepatocellular carcinoma, from 2001 we started to adapt our policies to the Milan criteria, and our results are acceptable and comparable to the worldwide results. The 5-year survival after OLT for HCC is more than 50\% comparing to the more than $80 \% 5$-year survival rate after OLT for other indications [6]. In this review the author analyzes the survival results of an ongoing study of liver transplantation for hepatocellular carcinoma between 1 January 2004 and 1 January 2011. Our aim is to find the possible ways to improve patient survival by refining recipient selection and tailoring individual immunosuppressive therapy.

\section{Material and method}

A single-institution study of 29 consecutive adult patients undergoing orthotopic liver transplantation between 1 January 2004 and 1 January 2011 with the indication of hepatocellular carcinoma. Survival was calculated with Kaplan-Meyer log rank analysis. Mean age at time of transplantation was 50.8 years (28-63). The median follow-up was 1236.3 days (89-2266). $55.17 \%$ of the recipients were male $(n=16)$ and $44.82 \%$ were female $(n=13)$. All surgeons used similar "cross-clamp" technique (recipient hepatectomy including vena cava segment). The histological examinations are under investigation regarding tumour 


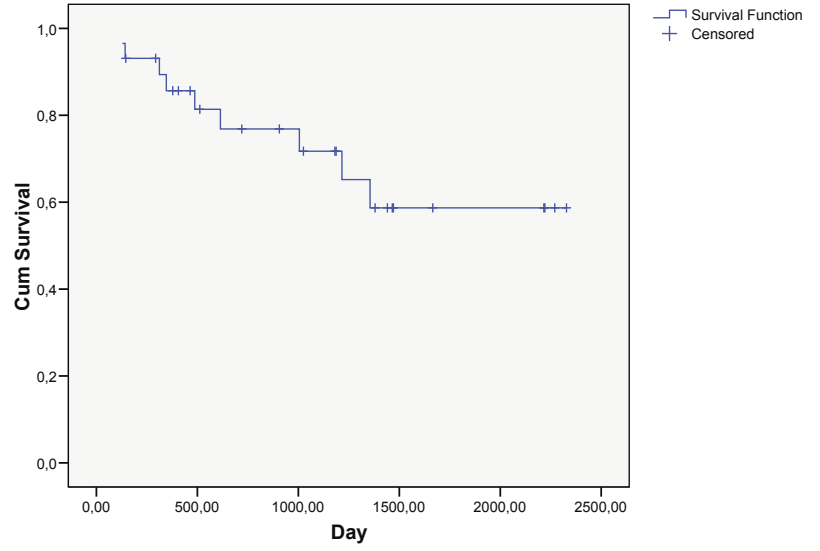

Fig. 1. Survival after liver transplantation for hepatocellular carcinoma in the Department of Transplantation and Surgery, Faculty of Medicine, Semmelweis University, Budapest, Hungary, 2004-2010.

size, grade, vessel invasion and extrahepatic propagation. The average intraoperative demand for blood transfusion was 5.8 units (0-26). All of the liver transplant recipients received a maintenance immunosuppressive therapy combination of tacrolimus or cyclosporine A, mycophenolatemofetil (MMF) and corticosteroids. In the case of tumour recurrency, the immunosuppresion was converted into an mTOR-inhibitor (sirolimus or everolimus).

\section{Results}

Survival was calculated with Kaplan-Meyer log rank analysis (Figure 1). Nine of our patients died in the late postoperative period (31.0\%). The main cause of death was HCC recurrency, in 6 cases: 3 in the liver, 1 in the lung, 1 in the cardia and 1 in the femur were observed. 2 patients died because of HCV recurrency and 1 with liver angiosarcoma. The 1 -year survival was $85.7 \%$ and the 3 -year survival was $71.7 \%$. According to the histology fifteen patients were within the Milan criteria and 14 exceeded the criteria.

\section{Discussion}

While patients with early resectable HCC and preserved hepatic function should undergo surgical resection, in those with unresectable disease due to underlying liver dysfunction, orthotopic liver transplantation offers the best chance for cure. Liver transplantation for hepatic tumours is known as a proven treatment opportunity. The first results were unsatisfactory and showed that only a highly selected patient population would benefit from transplantation. According to the United Network for Organ Sharing database, the 5-year survival of liver transplantation for hepatocellular carcinoma (HCC) was only $25 \%$ in the end of the 1980's, but the outcome has improved over the past decades because of the appropriate recipient selection. [7] According to Hoti et al. [8], currently, 15\% of all liver transplants are performed for hepatocellular carcinoma. Liver transplantation for HCC in the adult population ends in good results for patients whose tumour masses do not exceed the Milan criteria. In patients with small HCC at an early stage and preserved liver function, liver resection is an alternative method. Liver resection may present similar short term survival results to orthotopic liver transplantation (OLT), and avoids the long-term adverse effects of immunosuppression. However, liver transplantation is associated with longer disease-free survival in well-compensated cirrhotic patients with early stage hepatocellular carcinoma [9].

Cholangiocarcinoma treated by aggressive combination of chemo- and radiotherapy after transplantation showes lower, but promising results. Results of OLT are excellent in paediatric patients with unresecable and/or recurrent hepatoblastoma. Epithelioid hemangioendothelioma is also an indication, even in the presence of extrahepatic propagation. Angiosarcoma is associated with very poor survival and considered as a contraindication. Liver transplantation for metastatic liver disease from neuroendocrine tumours can result in long-term survival. Transplantation for colorectal liver metastases is currently contraindicated after the poor outcomes in the 1990's, but requires further re-evaluation according to the overall improvement in liver transplantation by at least $20 \%$ [10].

The treatment of hepatocellular carcinoma by liver transplantation has changed significantly in the last two decades. Introduction of measure criteria - such as the $\mathrm{Mi}$ lan or UCSF-expanded criteria - and the proper recipient selection have led to satisfying survival, but it is necessary to achieve better results. The role of interventional radiological methods, such as chemoembolisation and radiofrequency ablation in bridging and down-staging modalities is prominent $[11,12]$.

Expanding the HCC criteria for OLT allows more recipients to be eligible for transplantation, but there are also arguments against it because of the increased risk of vascular invasion and tumour recurrence at higher stages of HCC. The most important limitation of OLT for HCC is the shortage of deceased donors and so the liver grafts, and the development of living donor liver transplantation could be a solution for this donor shortage [13].

Our results with the 1- and 3-year survival rates $85.7 \%$ and $71.7 \%$ respectively - are comparable to other's in the literature. Herrero et al. [14] reported a 1-, 3-, 5-, 7-, and 10-year survival rates of their 73 transplanted HCC patients were $86 \%, 74 \%, 70 \%, 61 \%$, and $50 \%$, respectively. Similarly their patients exceeding Milan criteria had a higher dropout rate due to tumoral progression. According to a large multicenter study by Mazzaferro et al. [15] for those transplanted outside the Milan criteria, 5-year overall survival was $53.6 \%$ compared with $73.3 \%$ for those that met the criteria.

Another very important issue is the effective immunosuppression therapy by using drugs that help to decrease HCC recurrence. Introduction of mTOR-inhibitors to the immunosuppressive therapy protocols guided to lower tumour incidence among transplant patients in general [16]. Coadministration of sorafenib and an mTOR in- 
hibitor could be effective in patients with posttransplant HCC recurrence not suitable for radical therapy, but the toxicity and efficacy need to be further evaluated [17]. In our department we are trying to find mTOR activity in the HCC, normal and cirrhotic liver biopsies to evaluate the potential efficacy of our immunosuppressive strategy. Asian transplant centers have significantly modified the previous restrictions with the use of biomarker des-carboxy-prothrombin [18]. These strategies should help to improve better outcomes of liver transplantation for malignancies in the future.

\section{Conclusion}

Further investigations are needed on the field of histological and biological attributes of the hepatocellular carcinoma. Our aim is to find a better and individualized immunosuppressive protocol, wich is not only protective against acute rejection of the transplanted organ, but also protective against recurrency or de novo manifestation of malignancy. Moreover it is important to expand the liver donor pool by developing the living donor liver transplant program.

\section{References}

1. Adam R, Hoti E. Semin - Liver transplantation: the current situation. Liver Dis. 2009 Feb;29(1):3-18.

2. Befeler AS, Hayashi PH, Di Bisceglie AM. Liver transplantation for hepatocellular carcinoma. Gastroenterology. 2005;128:1752-1764.

3. Ringe B, Wittekind C, Bechstein WO, Bunzendahl H, Pichlmayr R. The role of liver transplantation in hepatobiliary malignancy: a retrospective analysis of 95 patients with particular regard to tumor stage and recurrence. Ann Surg. 1989;209:88-98.
4. Mazzaferro $V$, Regalia E, Doci $R$, et al. Liver transplantation for the treatment of small hepatocellular carcinomas in patients with cirrhosis. $\mathrm{N}$ Engl J Med. 1996;334:693-699.

5. Yao FY, Kinkhabwala M, LaBerge JM, et al. The impact of pre-operative loco-regional therapy on outcome after liver transplantation for hepatocellular carcinoma. Am J Transplant 2005;5:795-804.

6. Fehérvári I. Hepatocellular carcinoma and liver transplantation. Orv Hetil. 2010 Aug 8;151(32):1285-8. Hungarian.

7. Yoo HY, Patt CH, Geschwind JF, Thuluvath PJ. The outcome of liver transplantation in patients with hepatocellular carcinoma in the United States between 1988 and 2001: 5-year survival has improved significantly with time. J. Clin. Oncol. 2003;21:4329-4335.

8. Hoti E, Adam R. Liver transplantation for primary and metastatic liver cancers. Transpl Int. 2008 Dec;21(12):1107-17.

9. Bellavance EC, Lumpkins KM, Mentha G, et al. Surgical management of early-stage hepatocellular carcinoma: resection or transplantation? J Gastrointest Surg. 2008 Oct;12(10):1699-708.

10. Fehérvári I. Májátültetés. Gastro Update 2009: 294 Hungarian.

11. Heckman JT, et al. Bridging locoregional therapy for hepatocellular carcinoma prior to liver transplantation. Ann. Surgical Oncology 2008. vol15(11):3169-3177

12. Chapman WC, et al. Outcomes of neoadjuvant transarterial chemoembolization to downstage hepatocellular carcinoma before liver transplantation. Ann. Surgery 2008.vol 248(4):617-625.

13. Mazzaferro V, Chun YS, Poon RT, et al. Liver transplantation for hepatocellular carcinoma. Ann Surg Oncol. 2008 Apr;15(4):1001-7.

14. Herrero JI, Sangro B, Pardo F, et al. Liver transplantation in patients with hepatocellular carcinoma across Milan criteria. Liver Transpl. 2008 Mar;14(3):272-8.

15. Mazzaferro V, Llovet JM, Miceli R, et al; Metroticket Investigator Study Group. Predicting survival after liver transplantation in patients with hepatocellular carcinoma beyond the Milan criteria: a retrospective, exploratory analysis. Lancet Oncol. 2009 Jan;10(1):35-43.

16. Vivarelli M, Dazzi A, Cucchetti A, et al. Sirolimus in liver transplant recipients: a large single-center experience. Transplant Proc. 2010 Sep;42(7):2579-84.

17. Gomez-Martin C, Bustamante J, Castroagudin JF, et al. Efficacy and safety of sorafenib in combination with mammalian target of rapamycin inhibitors for recurrent hepatocellular carcinoma after liver transplantation. Liver Transpl. 2012 Jan;18(1):45-52.

18. Ito T, Takada $Y$, Ueda M, et al. Expansion of selection criteria for patients with hepatocellular carcinoma in living donor liver transplantation. Liver Transpl. 2007;13:1637-1644. 